\title{
Elastic imaging of subsurface Structure with Equivalent Offset Migration for multicomponent seismic data
}

\author{
Tomoaki TANAKA $^{1}$, Hitoshi MIKADA ${ }^{1}$ and Junichi TAKEKAWA ${ }^{1}$ \\ ${ }^{1}$ Dept. of Civil and Earth Res. Eng., Kyoto University
}

\begin{abstract}
Equivalent Offset Migration (EOM) was proposed to have both advantages of the conventional post-stack processing and velocity analysis as an alternative method to partial prestack migration, and draw attention in exploration geophysics for its computational efficiency and imaging accuracy. In the conventional EOM, it is mainly to use the vertical component of received waveforms, not horizontal components. However, it is necessary to get S-wave velocity structure in order to establish the sub-surface model including petrophysical properties. Thus, we conduct numerical experiments to verify the possibility of extracting information about S-wave velocity structure using EOM with the horizontal components. Our numerical results show that EOM based on the horizontal components can increase the amount of information of S-wave velocity whereas some unique difficulties to the horizontal components should be addressed.
\end{abstract}

\section{INTRODUCTION}

Seismic reflection survey is one of the most efficient methods for exploring subsurface natural resources, such as oil or natural gas reservoirs. Although conventional reflection imaging methods using poststack time migration work well for horizontal multi-layered structure, it is difficult to apply the conventional techniques to image complex subsurface structure. Prestack depth migration could be used to image such complex structure, but requires precise seismic velocity models with enough accuracy. Partial prestack migration is therefore used to estimate velocities as a trial-and-error method with the conventional post-stack processing methods. On the other hand, equivalent offset migration ${ }^{1)}(\mathrm{EOM})$ was proposed to exploit both advantages of the conventional post-stack processing and velocity analysis even for complex subsurface structure (Bancroft et al., 1998) as an alternative method to partial prestack migration, and draw attention in exploration geophysics for its computational efficiency and imaging accuracy.

In the conventional EOM, it is mainly to use the vertical component of received waveforms, not horizontal components. However, it is necessary to obtain S-wave velocity structure in order to establish the sub-surface model including petrophysical properties. We would like not to employ EOM to take the advantages of the processing efficiency but to extend it to use the horizontal components of waveforms for petrophysical analysis.

\section{METHOD}

EOM is the prestack time migration method for seismic reflection survey waveform data and "it is based on Kirchhoff prestack time migration ${ }^{2), 11}$.

In Fig. 1, $h$ is half offset, SP is a point like an epicenter against scatter point (SP), $x$ is the distance between SP and Mid Point, $t_{0}$ is a two-way travel time in zero offset section at SP. Considering the geometry of Fig. 1, two-way travel time $t$ is shown,

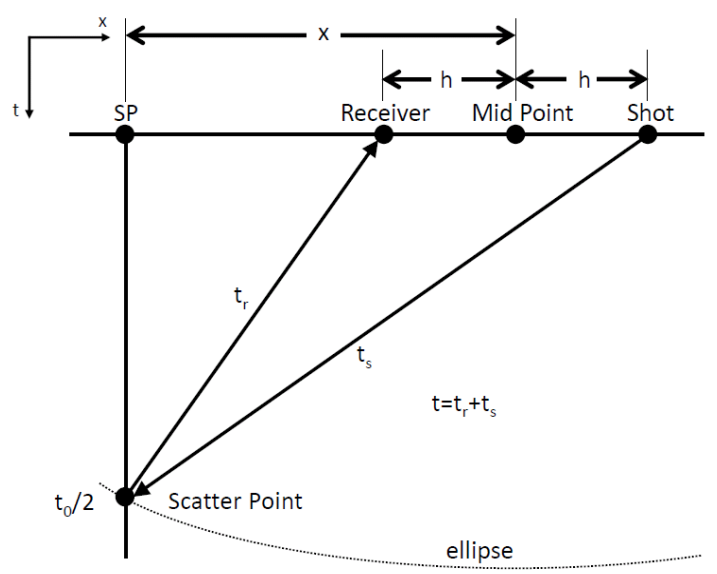

Fig. 1 Geometry in Kirchhoff Migration 


$$
\begin{gathered}
t=t_{s}+t_{r} \\
t=\left[\left(\frac{t_{0}}{2}\right)^{2}+\frac{(x+h)^{2}}{V_{m i g}^{2}}\right]^{1 / 2}+\left[\left(\frac{t_{0}}{2}\right)^{2}+\frac{(x-h)^{2}}{V_{m i g}^{2}}\right]^{1 / 2}
\end{gathered}
$$

with $V_{\text {mig }}$, migration velocity; $t_{s}, t_{r}$, one-way travel time from shot to $(\mathrm{SP})$, from $(\mathrm{SP})$ to receiver.

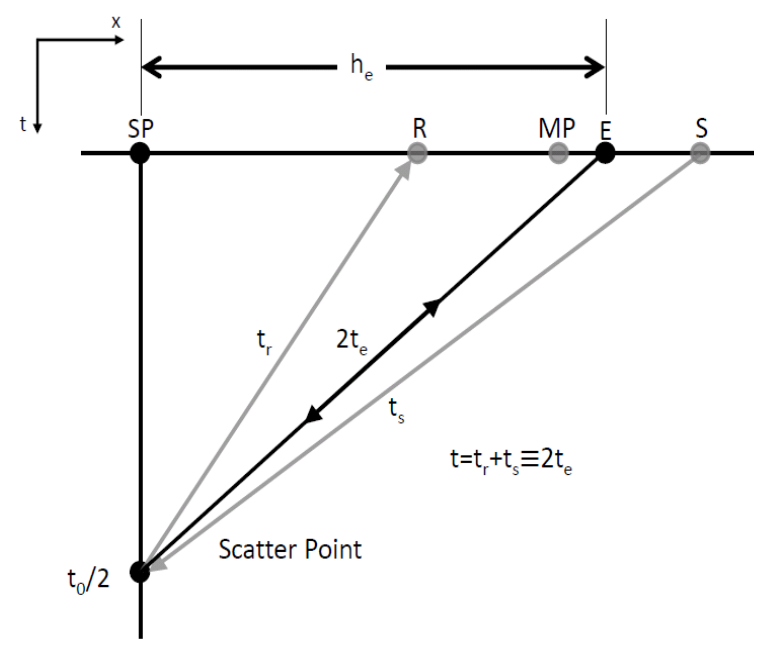

Fig. 2 Geometry in EOM

In Fig. 2, $\mathrm{E}$ is one of the zero offset points to the (SP) with the same two-way travel time: $t_{e}$, and $h_{e}$ is the distance between SP and E. According to Fig 2, we get several equations, (3)-(6).

$$
2 t_{e} \equiv t_{s}+t_{r}=t
$$

$2\left[\left(\frac{t_{0}}{2}\right)^{2}+\frac{h_{e}^{2}}{V_{m i g}^{2}}\right]^{\frac{1}{2}}$

$$
\begin{gathered}
=\left[\left(\frac{t_{0}}{2}\right)^{2}+\frac{(x+h)^{2}}{V_{\text {mig }}^{2}}\right]^{1 / 2}+\left[\left(\frac{t_{0}}{2}\right)^{2}+\frac{(x-h)^{2}}{V_{\text {mig }}^{2}}\right]^{1 / 2} \\
h_{e}^{2}=x^{2}+h^{2}-\left(\frac{2 x h}{t V_{\text {mig }}}\right)^{2} \\
\left(\frac{t V_{\text {mig }}}{2}\right)^{2}-\left(\frac{t_{0} V_{\text {mig }}}{2}\right)^{2}=h_{e}^{2}
\end{gathered}
$$

Using eq. (5), input data for the particular (SP) are migrated on hyperbola as Fig. 3, and each data set at respective SP is common scatter point (CSP) gather. Equation (6) is used for normal moveout (NMO) of CSP gathers.

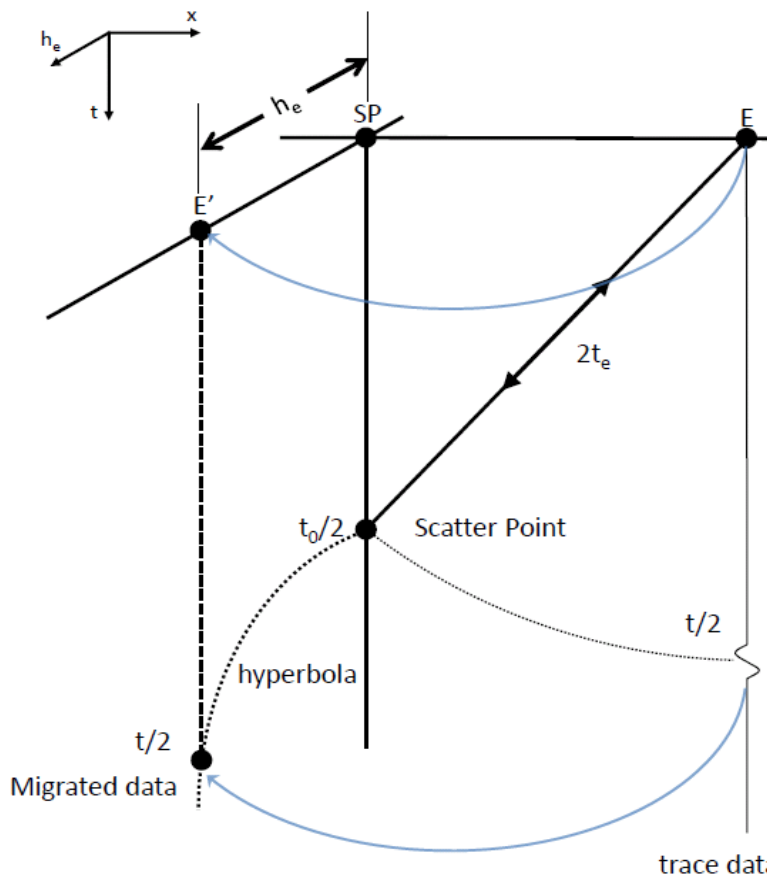

Fig. 3 Making CSP gather

\section{NUMERICAL MODELS}

In our study, we simulate 2-D seismic wave propagation using finite difference method with staggered $\operatorname{grid}^{3)}$.

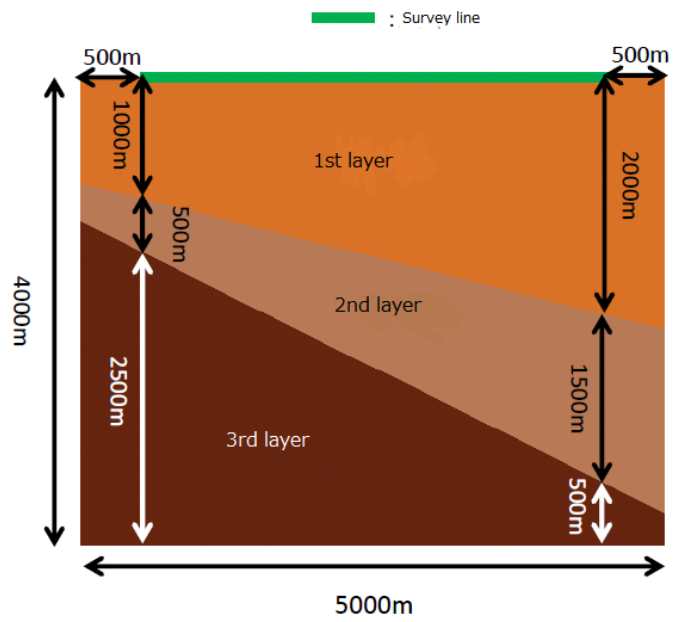

Fig. 4 2-D numerical elastic subsurface model

In Fig. 4, we construct a 2-D elastic model and put 201 sources and 201 receivers on the surface survey line with $20 \mathrm{~m}$ intervals. Each source is applied as a vertical force and its function is Ricker wavelet whose dominant frequency is $10 \mathrm{~Hz}$. The upper boundary condition of the model is free boundary condition and others are C-PML boundary condition $^{4)}$. The physical properties of the numerical model are shown in Table 1 and Table 2. 
Table 1 Density and velocity structure

\begin{tabular}{|c|c|c|c|}
\hline & $\begin{array}{c}\text { Density } \\
\left(\mathrm{g} / \mathrm{cm}^{3}\right)\end{array}$ & $\begin{array}{c}\text { P-wave vel. } \\
(\mathrm{m} / \mathrm{sec})\end{array}$ & $\begin{array}{c}\text { S-wave vel. } \\
(\mathrm{m} / \mathrm{sec})\end{array}$ \\
\hline 1st layer & 2.50 & 3000 & 1732 \\
\hline 2nd layer & 2.70 & 3200 & 1847 \\
\hline 3rd layer & 2.80 & 4000 & 2309 \\
\hline
\end{tabular}

Table 2 Parameters

\begin{tabular}{|c|c|}
\hline Total number of grids & $501 \times 401$ \\
Thickness of C-PML & 20 grids \\
Timestep interval & $5.0 \times 10^{-4}(\mathrm{sec})$ \\
Total simulation time & $4.0(\mathrm{sec})$ \\
Grid interval & $10 \mathrm{~m}$ \\
Source, Receiver CSP interval & Respectively $20 \mathrm{~m}$ \\
$\begin{array}{c}\text { The number of Source, } \\
\text { Receiver, CSP }\end{array}$ & Respectively201 点 \\
$\begin{array}{c}\text { Dominant frequency } \\
\text { (Ricker wavelet) }\end{array}$ & $10(\mathrm{~Hz})$ \\
\hline
\end{tabular}

\section{DATA PROCESSING}

The flow chart is shown in Fig. 5. We use horizontal waveform data in order to obtain S-wave velocity information. As making CSP gathers, we use amplitude scaling ${ }^{5)}$ whose equation is denoted below (eq. (7)).

$$
\text { scale }=1-\frac{x}{h_{e}}
$$

Simulating 2-D wave propagation to get horizontal waveform

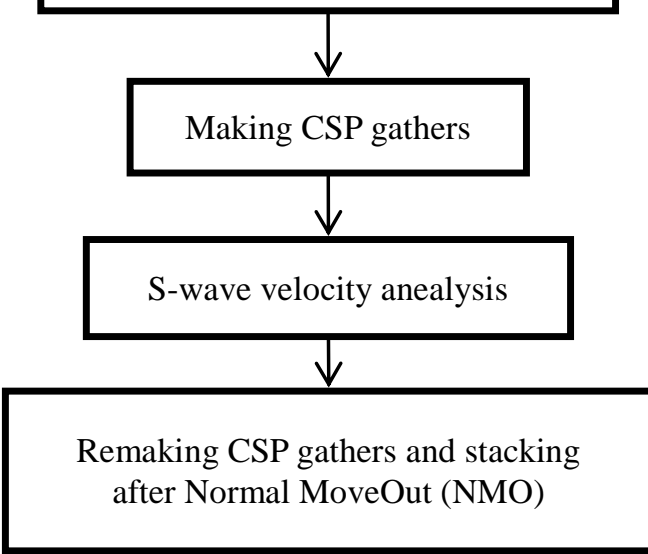

Fig. 5 EOM flow chart in our study

\section{RESULTS}

The CSP gather is shown in Fig. 6. Several points where the wave amplitude is flipped can be observed.

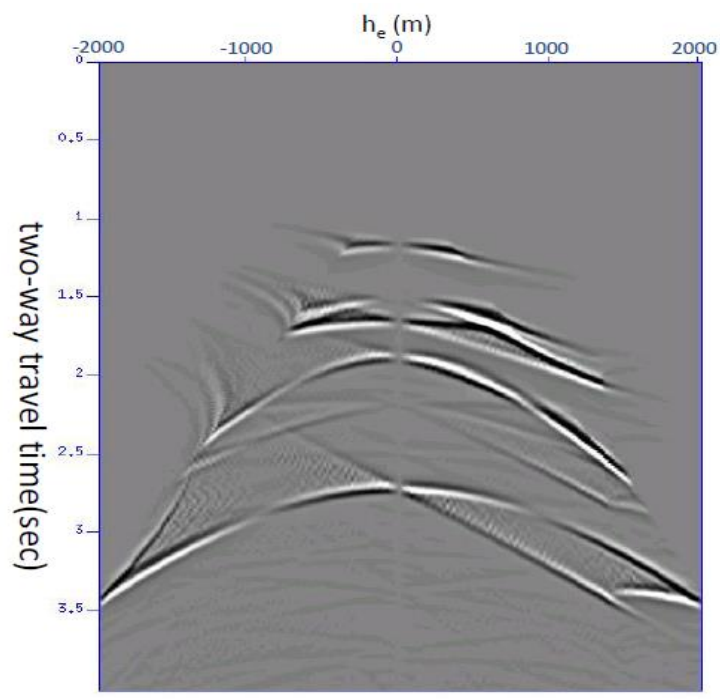

Fig. 6 CSP gather at CSP101

Neighbor the point " $h_{e}=0$ ", the signs of right side waveform and left side are inverted. Consequently, in case of $h_{e}$ under zero, we reverse the sign of waveform and stack gather data to make zero offset section. The zero offset section is shown in Fig.7. We can observe several inclined reflection surfaces with different dips. Reflection events appeared around $2 \mathrm{~s}-2.5 \mathrm{~s}$ and $3 \mathrm{~s}-3.5 \mathrm{~s}$ are S-S events, whereas other events stem from P-P, P-S and S-P. S-S event would be emphasized by considering flipped points in Fig.6.

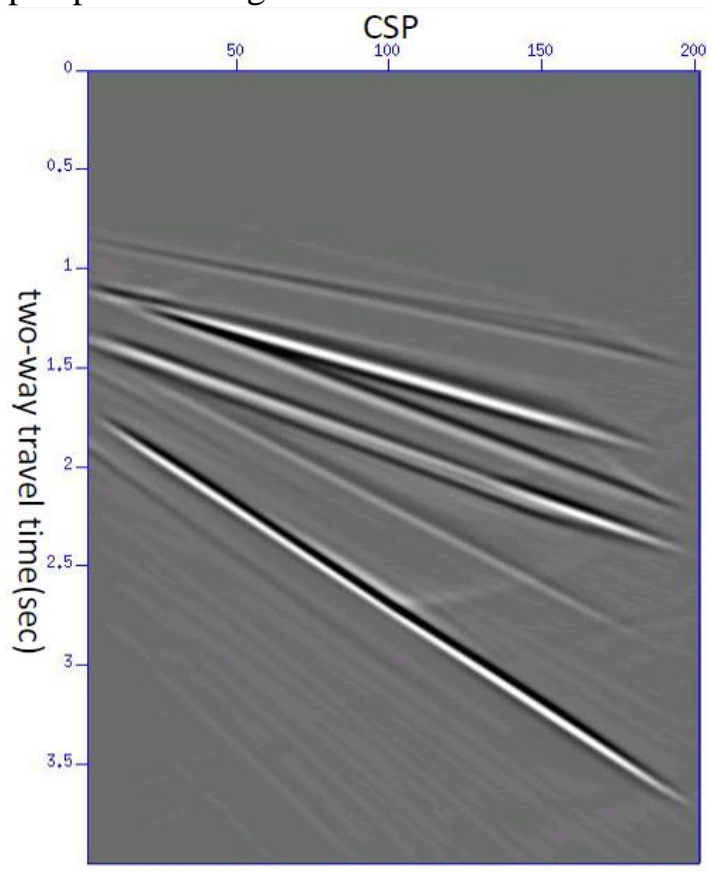

Fig. 7 Zero offset section 


\section{CONCLUSION}

In our study, we conduct EOM with horizontal component waveform in order to obtain $\mathrm{S}$-wave velocity information and we can get zero offset section with enough accuracy using EOM by horizontal component. We would like to study about waveform flipped points in CSP gather for further improvement of imaging results.

ACKNOWLEDGEMENT: We would like to thank past and present menbers of our laboratory.

\section{REFERENCES}

1) Bancroft, J.C., Geiger, H. D., and Margrave, G. F., 1998, The equivalent offset method of prestack time migration, Geophysics, 63, 2042-2053.

2) Schneider, W., 1978, Integral formulation for migration in two and three dimensions, Geophysics, 43, 49-76.

3) Virieux, J., 1986, P-SV wave propagation in heterogeneous media: Velocity-stress finite-difference method, Geophysics, 51, 889-901.

4) Komatitsch, D., and Martin, R., 2007, An unsplit convolutional perfectly matched layer improved at grazing incidence for the seismic wave equation, Geophysics, 72, 155-167.

5) Li, X., Xu, Y., and Bancroft, J. C., 1997, Equivalent offset migration: the implementation and application update, CREWES Project Research Report, 9, 27.1-27.11. 